\title{
Klebsiella pneumoniae-Induced Multiple Infections in a Diabetes Mellitus Patient: Pneumonia, Liver Abscess, Endogenous Endophthalmitis, Urinary Tract Infection
}

\author{
Meng-Yu Wu ${ }^{1,2}\left(\mathbb{D}\right.$, , Yueh-Tseng Hou ${ }^{1,2}$ and Giou-Teng Yiang ${ }^{1,2, *}$ \\ 1 Department of Emergency Medicine, Taipei Tzu Chi Hospital, Buddhist Tzu Chi Medical Foundation, \\ New Taipei 231, Taiwan; skyshangrila@gmail.com (M.-Y.W.); brianann75@gmail.com (Y.-T.H.) \\ 2 Department of Emergency Medicine, School of Medicine, Tzu Chi University, Hualien 970, Taiwan \\ * Correspondence: gtyiang@gmail.com; Tel.: +886-2-6628-9779; Fax: +886-2-6628-9009
}

Received: 13 March 2018; Accepted: 28 March 2018; Published: 28 March 2018

\begin{abstract}
Klebsiella pneumoniae-induced endogenous endophthalmitis is a rare but devastating disease, which usually occurs in immunosuppressed patients. Poorly controlled diabetes mellitus is a significant risk factor for this disease. The most common distant infection in patients with K. pneumoniae-induced endogenous endophthalmitis was reported to be liver abscess. Other less commons distant infections were found to be catheter-related bloodstream infection, urinary tract infection, soft tissue infection, renal and psoas abscesses, and endocarditis. Patients with coexisting multiple infection sites are rare according to previous studies. These patients may have a high risk of sepsis and death. Here, we present a case of a 64-year-old male who presented with a progressive visual disturbance with general malaise. A K. pneumoniae-induced endogenous endophthalmitis was suspected. After surveying distant infection sites, pneumonia, liver abscess, and urinary tract infection were reported. This paper also describes the clinical features of endogenous endophthalmitis and highlights K. pneumoniae-induced multiple infections in a diabetes mellitus patient, thus providing information that will be useful for physicians to perform timely interventions and avoid sepsis.
\end{abstract}

Keywords: Klebsiella pneumoniae; endogenous endophthalmitis; liver abscess; diabetes mellitus; sepsis

\section{Introduction}

Endogenous endophthalmitis, also known as metastatic endophthalmitis, is a rare but dangerous endophthalmitis resulting in the occurrence of distant infections trhough hematogenous spread [1]. Most patients are immunosuppressed individuals, affected by diabetes mellitus, or intravenous drug abusers [2]. These patients have a high risk to develop bacteremia or sepsis. Klebsiella pneumonia is the common pathogen isolated from diabetes mellitus patients with endogenous endophthalmitis. In some patients, the K. pneumoniae-induced multiple infections may arise from gastrointestinal disease following bacterial translocation from the intestine to the portal circulation. To early diagnose and timely treat endogenous endophthalmitis, a broad examination is necessary. Surveying the primary site is also important. Thus, physicians need to be aware of the clinical features of endogenous endophthalmitis. Here, we present a case of a 64-year-old male who presented with progressive visual disturbance due to K. pneumoniae-induced multiple infections. This paper also describes the clinical features of endogenous endophthalmitis and highlights $K$. pneumoniae-induced multiple infections in a diabetes mellitus patient, thus providing information that will be useful for physicians to timely perform interventions and avoid sepsis. 


\section{Case Presentation Section}

A 64-year-old male presented with acute onset of progressive visual disturbance ongoing for $4-5$ days. There was no history of trauma. The blurry vision was progressively accompanied by poor appetite and general malaise. He denied any fever, chills, productive cough, chest pain, abdominal pain, diarrhea, frequency, urgency, and dysuria. His temperature was $35.6{ }^{\circ} \mathrm{C}$, the blood pressure was $121 / 80 \mathrm{mmHg}$, and the heart rate were $100 / \mathrm{min}$. An abdominal physical examination revealed no local tenderness, murphy sign, McBurney's point tenderness, or knocking pain. In addition, the pupil reflex at Oculus Dexter (OD) was characterized by a sluggish reaction. The relative afferent pupillary defect (RAPD) sign was negative. A keratin epithelial scar over the temporal region was noted in OD, associated with injected conjunctiva. The anterior chamber was deep and filled with cells, showing a grade 4+ at OD. Nuclear sclerosis was noted in Oculus Uterque (OU) lens. The fundus could not be approached because of poor visual field (Figure 1). The B-scan revealed vitreous opacity, indicating suspected hemorrhage or pus.

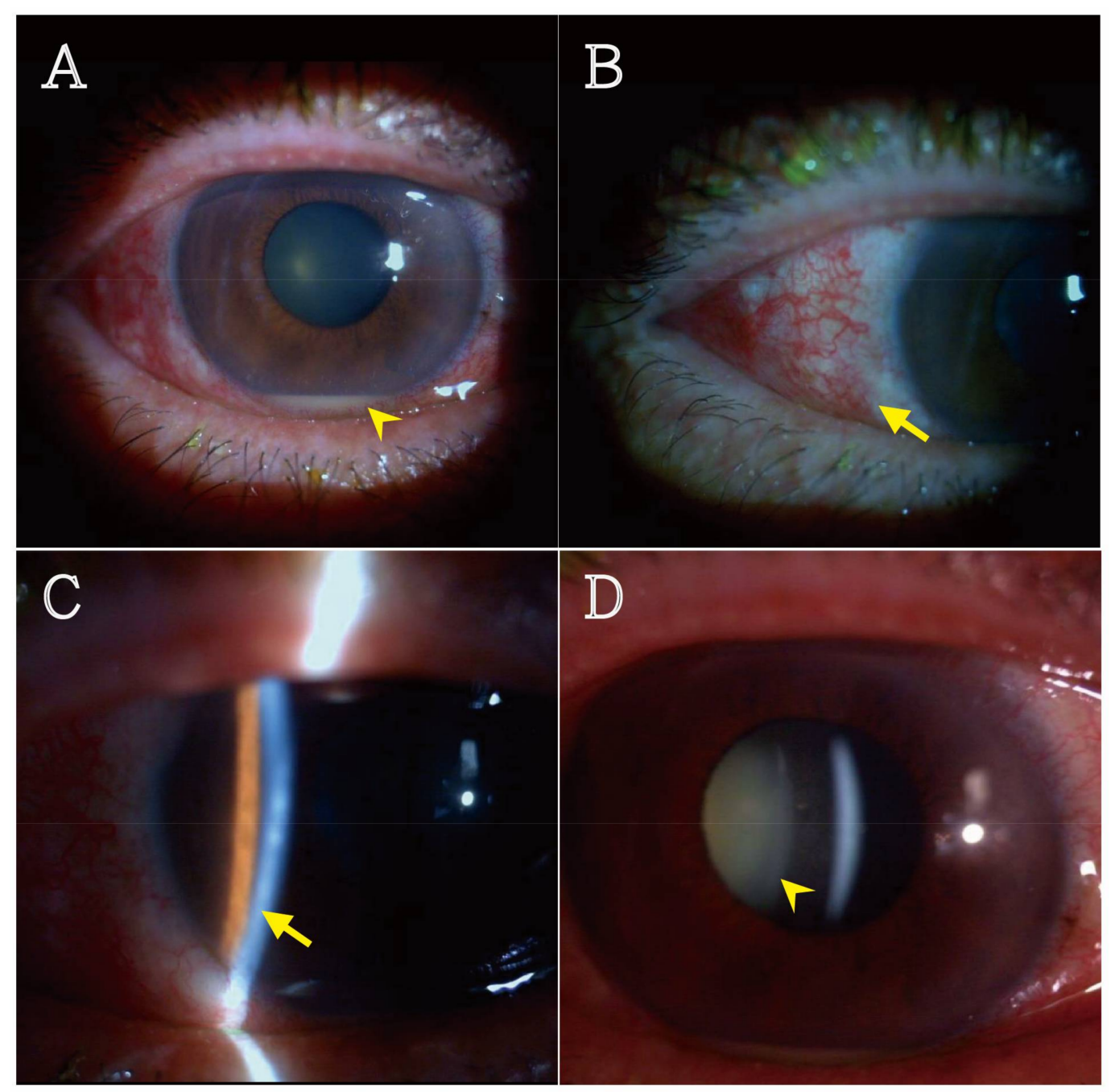

Figure 1. Clinical feature of the patient's right eye. (A) A hypopyon is shown (arrow head); (B) The conjunctiva was injected (arrow); (C) Severe accumulation of inflammatory cells in the anterior chamber (arrow); (D) Nuclear sclerosis is shown (arrow head).

A purulent endogenous endophalmitis was suspected. The poor appetite and general malaise could not be well explained by endophalmitis. To find potential additional infection sites, laboratory data were collected from this patient and revealed: white cell count 15,210 cells/ $\mu \mathrm{L}$ (band form 
neutrophils $6.0 \%$, segment form neutrophils $76.0 \%$, lymphocytes $12.0 \%$, eosinophils $0.0 \%$, and monocytes $6.0 \%$ ) (normal range: 3800 to 9800 cells $/ \mu \mathrm{L}$ ), hemoglobin $11.9 \mathrm{~g} / \mathrm{dL}$ (normal range: 13 to $18 \mathrm{~g} / \mathrm{dL}$ ), platelet counts 331,000 cells $/ \mathrm{mL}$ (normal range: 117,000 to $317,000 / \mathrm{mL}$ ), creatinine $1.0 \mathrm{mg} / \mathrm{dL}$ (normal range: 0.7 to $1.3 \mathrm{mg} / \mathrm{dL}$ ), aspartate aminotransferase $23 \mathrm{U} / \mathrm{L}$ (normal range: 13 to $63 \mathrm{U} / \mathrm{L}$ ), alanine aminotransferase $467 \mathrm{U} / \mathrm{L}$ (normal range: 14 to $59 \mathrm{U} / \mathrm{L}$ ), total bilirubin $0.80 \mathrm{mg} / \mathrm{dL}$ (normal range: 0.0 to $1.0 \mathrm{mg} / \mathrm{dL}$ ), direct bilirubin $0.33 \mathrm{mg} / \mathrm{dL}$ (normal range: 0.1 to $0.4 \mathrm{mg} / \mathrm{dL}$ ), albumin $1.9 \mathrm{~g} / \mathrm{dL}$ (normal range: 3.5 to $5.0 \mathrm{~g} / \mathrm{dL}$ ), glucose $336 \mathrm{mg} / \mathrm{dL}$ (normal range: 70 to $100 \mathrm{mg} / \mathrm{dL}$ ), Troponin I < $0.01 \mathrm{IU} / \mathrm{L}$ (normal range: $<0.01 \mathrm{IU} / \mathrm{L}$ ), and C-Reactive protein $16.44 \mathrm{mg} / \mathrm{L}$ (normal range: 0.0 to $0.33 \mathrm{mg} / \mathrm{L}$ ). Urinalysis results showed red cell count $0-2 / \mathrm{HPF}$ (high power field) and white cell count $5-10 / \mathrm{HPF}$. A chest $\mathrm{X}$-ray and an abdominal echo were arranged to rule out the presence of K. pneumoniae-induced liver abscess and pneumonia.

The chest X-ray revealed bilateral pulmonary infiltration with air bronchograms. The abdominal echo showed multiple poorly demarcated lesions with a predominantly hypoechoic appearance. Gas bubbles were seen in the liver lesions. Computed tomography (CT) confirmed the diagnosis of liver abscess (Figure 2). A brain CT was done and revealed no infection sign.
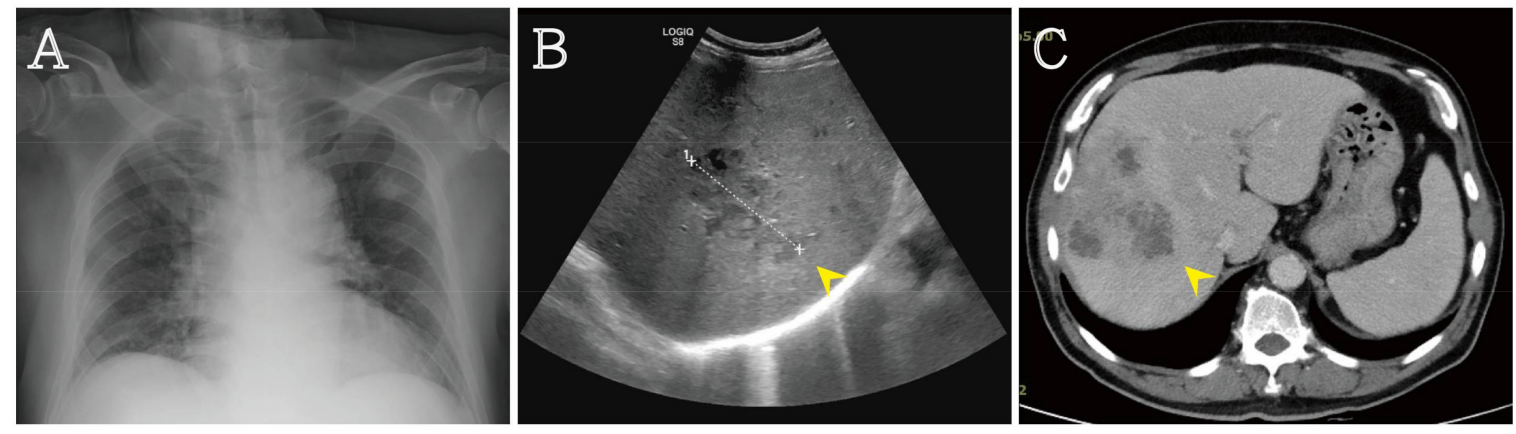

Figure 2. (A) X-ray exam revealing bilateral pulmonary infiltration with air bronchograms; (B) abdominal echo showing multiple hypoechoic lesions with gas bubbles (arrowhead); (C) Computed tomography exam revealing several heterogenous lesions in the liver (arrowhead).

The broad-spectrum antibiotic ceftriaxone was administrated with metronidazole to treat the infection. The culture of urine revealed K. pneumoniae, but the culture of blood and of eye discharge showed no specific bacteria growth. The sputum culture revealed mixed normal flora. The patient was diagnosed with K. pneumoniae-induced pneumonia, liver abscess, endogenous endophthalmitis, and urinary tract infection. After antibiotic administration while controlling blood sugar levels, the infection was under control, with a final OU visual acuity of 20/200. Once in a stable condition, the patient was received regularly for follow up at outpatient department. A written informed consent was obtained from the patient.

\section{Discussion}

Endogenous endophthalmitis, usually resulting from hematogenous spread of pathogens in immunosuppressed patient, is an important hint for physicians in the emergency department [2]. Endogenous endophthalmitis is rare, accounting for $2-8 \%$ of all cases of endophthalmitis [3,4]. Most cases of endogenous endophthalmitis are due to fungal infection and Gram-positive bacteremia. PP Connell et al. [5] reported that culture results of exams performed in a 10-year period at a tertiary referral center revealed fungal isolates representing $65.9 \%$ of the isolated microorganisms, Gram-negative bacteria representing 19.5\%, and Gram-positive bacteria representing $14.6 \%$. The culture positive rate was $64.1 \%$. Among Gram-negative organisms, K. pneumoniae accounted for $50 \%$ of total Gram-negative bacteria. In Asian countries, the K. pneumoniae-induced liver abscess is the major source of Gram-negative organisms. Recently, K. pneumoniae-induced liver abscess has been reported to be associated with K. pneumoniae $\mathrm{K} 1$ or K2 serotypes [6]. A similar result was also 
reported by Fung et al. [7] who found that K. pneumoniae serotype K1, which is a virulence factor, is common in Taiwan and South Africa and plays an important role in the formation of liver abscess and endophthalmitis. Therefore, bacteremia should be highly suspected in patients with endogenous endopthalmitis. In these patients, an attempt should be made to identify the infection source, such as pneumonia and liver abscess, especially K. pneumonia-induce liver abscess.

Patients with diabetes mellitus have a high risk of K. pneumoniae-induced endogenous endophthalmitis, especially patients with a liver abscess. The metastatic infection rate of K. pneumoniae from liver abscesses was reported to be 33.3\% [8]. The most common distant infection was reported by Todokoro D et al. [1] to be liver abscess was, followed by catheter-related bloodstream infection and infections at other sites, such as urinary tract infection, soft tissue infection, renal and psoas abscess, and endocarditis. However, coinfection cases were rare and resulted more dangerous. In our case, multiple infection sites were suspected as a consequence of K. pneumoniae presence in the patient. We believe that multiple infections may be induced via bacteremia. The original infection site may be the liver, which is reported as the major infection site. To prevent sepsis progression, a broad-spectrum antibiotic therapy was administrated to treat K. pneumoniae infection. However, the outcome of K. pneumoniae-induced endogenous endophthalmitis occurred despite early antibiotic intervention. In this article, we highlight that a diagnosis of endogenous endophthalmitis was made and an early surveying the of potential distant infection sites and a timely treatment were performed to avoid the occurrence of possible complications.

Acknowledgments: This work was not supported by any fund.

Author Contributions: Meng-Yu Wu wrote the paper and contributed to the organization of the figures; Yueh-Tseng Hou and Giou-Teng Yiang provided conceptual input; Giou-Teng Yiang proofread and organized the manuscript. All authors reviewed the final version of the manuscript.

Conflicts of Interest: The authors declare no conflict of interest.

\section{References}

1. Todokoro, D.; Mochizuki, K.; Nishida, T.; Eguchi, H.; Miyamoto, T.; Hattori, T.; Suzuki, T.; Inoue, T.; Nejima, R.; Hoshi, S.; et al. Isolates and Antibiotic Susceptibilities of Endogenous Bacterial Endophthalmitis: A Retrospective Multicenter Study in Japan. J. Infect. Chemother. 2008. [CrossRef] [PubMed]

2. Karama, E.M.; Willermain, F.; Janssens, X.; Claus, M.; Van den Wijngaert, S.; Wang, J.T.; Verougstraete, C.; Caspers, L. Endogenous Endophthalmitis Complicating Klebsiella Pneumoniae Liver Abscess in Europe: Case Report. Int. Ophthalmol. 2008, 28, 111-113. [CrossRef] [PubMed]

3. Soon-Phaik, C.; Jap, A. Endogenous Endophthalmitis. Curr. Opin. Ophthalmol. 2001, 12, 464-470.

4. Shrader, S.K.; Band, J.D.; Lauter, C.B.; Murphy, P. The Clinical Spectrum of Endophthalmitis: Incidence, Predisposing Factors, and Features Influencing Outcome. J. Infect. Dis. 1990, 162, 115-120. [CrossRef] [PubMed]

5. Connell, P.P.; O’Neill, E.C.; Fabinyi, D.; Islam, F.M.A.; Buttery, R.; McCombe, M.; Essex, R.W.; Roufail, E.; Clark, B.; Chiu, D.; et al. Endogenous Endophthalmitis: 10-Year Experience at a Tertiary Referral Centre. Eye 2011, 25, 66-72. [CrossRef] [PubMed]

6. Siu, L.K.; Yeh, K.-M.; Lin, J.-C.; Fung, C.-P.; Chang, F.-Y. Klebsiella Pneumoniae Liver Abscess: A New Invasive Syndrome. Lancet Infect. Dis. 2012, 12, 881-887. [CrossRef]

7. Fung, C.P.; Chang, F.Y.; Lee, S.C.; Hu, B.S.; Kuo, B.I.; Liu, C.Y.; Ho, M.; Siu, L.K. A Global Emerging Disease of Klebsiella Pneumoniae Liver Abscess: Is Serotype K1 an Important Factor for Complicated Endophthalmitis? Gut 2002, 50, 420-424. [CrossRef] [PubMed]

8. Chang, Z.; Zheng, J.; Ma, Y.; Liu, Z. Analysis of Clinical and Ct Characteristics of Patients with Klebsiella Pneumoniae Liver Abscesses: An Insight into Risk Factors of Metastatic Infection. Int. J. Infect. Dis. 2015, 33, 50-54. [CrossRef] [PubMed]

(C) 2018 by the authors. Licensee MDPI, Basel, Switzerland. This article is an open access article distributed under the terms and conditions of the Creative Commons Attribution (CC BY) license (http://creativecommons.org/licenses/by/4.0/). 